\title{
Production of massless charm jets in pp collisions at next-to-leading order of QCD
}

\author{
Isabella Bierenbaum* and Gustav Krament \\ II. Institut für Theoretische Physik, Universität Hamburg, \\ Luruper Chaussee 149, 22761 Hamburg, Germany
}

(Dated: December 18, 2014)

\begin{abstract}
We present predictions for the inclusive production of charm jets in proton-proton collisions at $7 \mathrm{TeV}$. Several CTEQ parton distribution functions (PDFs) of the CTEQ6.6M type are employed, where two of the CTEQ6.6 PDFs have intrinsic charm. At large enough jet transverse momentum and large jet rapidity, the intrinsic charm content can be tested.
\end{abstract}

*Electronic address: isabella.bierenbaum@desy.de; Current address: Institutes of Physics and Mathematics, Humboldt-Universität zu Berlin, Unter den Linden 6, 10099 Berlin, Germany

†Electronic address: gustav.kramer@desy.de 


\section{INTRODUCTION}

The cross section for producing heavy quarks in proton-proton collisions at the Large Hadron Collider (LHC) can be computed in QCD. Calculations have been carried out at next-to-leading order (NLO) in perturbation theory [1]. So far, most of the predictions for charm-quark jets have been done in the massive quark scheme or fixed-flavor-number scheme (FFNS) in which charm quarks appear in the final state only and not as partons in the initial state, as for example in the zero-mass variable-flavor-number scheme (ZM-VFNS). The treatment of the charm quark being massless is justified as long as the transverse momentum $p_{T}$ of the produced jets is large enough, i.e. for $p_{T}^{2} \gg m^{2}$, where $m$ is the charmquark mass. In such a case, one might hope to obtain information on the charm content of the proton parton distribution function (PDF) by measuring cross sections in pp collisions for the production of charm jets at large $p_{T}$. Such a task has been pursued in connection with dijet photoproduction of charm jets by M. Klasen and one of us in order to get information on the photon PDF [2]. Another way to investigate the charm PDFs is to measure, instead of charm jets, the production of charmed hadrons, as for example the various kinds of D mesons or charmed baryons. This has been considered in several publications on the production of $\mathrm{D}$ mesons in photoproduction and hadron-hadron collisions in the ZM-VFNS [3], 4] and the general-mass variable-flavor-number scheme (GM-VFNS) [5]. The GM-VFNS incorporates finite charm-mass corrections taken from the FFNS in order to improve the predictions at small and medium $p_{T}$ s. This approach has already been used to predict single D-meson inclusive cross sections for various LHC experiments [6], which are found in good agreement with the experimental data [7-9]. Of course, these cross sections depend on the fragmentation functions for the fragmentation of the final state partons into the respective charmed mesons D or charmed baryons which are the result of fits to production cross sections at $e^{+} e^{-}$colliders. On the other hand, the charm-jet production cross sections do not depend on such fragmentation functions. Thus we can expect to obtain complementary information on the charm PDFs to the charmed-hadron cross sections, independent of the fragmentation functions input. The only requirement, however, is that the selection of charmed jets in the measurement must correspond to the selection in the theoretical cross section calculations. In the next section, we shall describe the theoretical framework and outline the input in terms of PDFs for the initial state. Section 3 contains our results for the 
charm-jet cross section. In this section, we also show a comparison with the single-inclusive jet cross section measured by the CMS collaboration at the LHC [10] in order to demonstrate that our jet cross section routine agrees with the experimental data with no special flavor selection. Similar data are also obtained by the ATLAS collaboration at the LHC [11]. A summary and our conclusions are presented in Section 4.

\section{THEORETICAL FRAMEWORK AND PDF INPUT}

For our calculation, we rely on previous work on dijet production in the reaction $\gamma+p \rightarrow$ jet $+X$ [12], in which cross sections for inclusive one-jet and two-jet production up to NLO for both the direct and the resolved contributions are calculated (for a review see [13]). The predictions of this work have been tested by many experimental studies of the H1 and ZEUS collaboration at HERA. The resolved part of this cross section routine can be used for pp collisions replacing the photon PDF by the proton PDF. Recent comparisons of the Tevatron and LHC jet data are usually performed with predictions of the NLOJET ++ routine [14] within the framework of FASTNLO [15]. The routine [12] contains quarks of all flavors and the gluon. For our purposes, it has been restricted to the case that at least one charm quark appears in the final state. In the initial state we have the contributions $c g, \bar{c} g, c q, \bar{c} q, c \bar{c}, c c$, $\bar{c} \bar{c}$, where $q$ is a light quark (or antiquark), with the restriction that $c \bar{c} \rightarrow g g$ and $c \bar{c} \rightarrow q \bar{q}$ in leading order (LO) and the corresponding contributions in NLO are removed, i.e. only terms like $c \bar{c} \rightarrow c \bar{c}$ and $c c \rightarrow c c, \bar{c} \bar{c} \rightarrow \bar{c} \bar{c}$ respectively, are retained. In addition, there are also contributions with light quarks and gluons in the initial state, as for example $q \bar{q} \rightarrow c \bar{c}$ and $g g \rightarrow c \bar{c}$, as well as the corresponding NLO contributions.

For our predictions, we employ various PDFs of the proton. For the inclusive jet cross section containing all flavors and to be compared with CMS measurements [10], we use the CTEQ CT10 version [16] as used in the CMS publication [10] with $n_{f}=5$, i.e. we include all flavors up to including the bottom quark. The asymptotic scale parameter is $\Lambda_{\overline{M S}}^{(5)}=0.262$ $\mathrm{GeV}$ corresponding to $\alpha_{s}^{5}\left(m_{Z}\right)=0.118$. We choose the renormalization scale $\mu_{R}=\xi_{R} p_{T}$ and the factorization scale $\mu_{F}=\xi_{F} p_{T}$, where $p_{T}$ is the largest transverse momentum of the two (or three) final state jets. $\xi_{R}$ and $\xi_{F}$ are dimensionless scale factors, which are varied about their default values $\xi_{R}=\xi_{F}=1$ to be specified later. The center-of-mass (c.m.) energies

of the proton in all calculations are taken as $\sqrt{S}=7 \mathrm{TeV}$, as for the data of CMS [10]. 
The bin size in $p_{T}$ is taken according to the CMS publication [10] and the rapidity interval is $-0.5 \leq y \leq 0.5$ as given in one of the curves in [10]. For the charm-jet cross sections, we concentrate our calculations on the large $p_{T}$ region, $p_{T} \geq 37 \mathrm{GeV}$. The bin sizes in $p_{T}$ and two of the rapidity bins $(|y| \leq 0.5$ and $2.0 \leq|y| \leq 2.2)$ for our charm-jet calculation are chosen in accordance with the CMS publication for inclusive b-jet production [17] since there are no data for inclusive c-jet production yet. In addition, we select as a third rapidity interval: $2.2 \leq|y| \leq 3.2$. The charm-jet calculations are done with the CTEQ6.6M PDF sets [18] and $n_{f}$ is taken to be $n_{f}=4$. The corresponding $\alpha_{s}$ is calculated with $n_{f}=4$ as well, with the $\Lambda$ value corresponding to this $n_{f}$ value. In the theoretical calculations, jets were reconstructed with the $k_{T}$-cluster algorithm in the longitudinally invariant inclusive mode [19]. The measurements of the inclusive jet cross sections are done with the anti- $k_{T}$ jet clustering algorithm [20] using as the distance parameter $\mathrm{R}=0.5$. This value for $\mathrm{R}$ is also applied for all the other calculations in this work. At $O\left(\alpha_{s}^{2}\right)$, the parton-level predictions from the $k_{T}$ and anti- $k_{T}$ algorithms are identical.

\section{RESULTS}

First we show our prediction of $d \sigma / d p_{T}$ for the $p+p \rightarrow$ single $j e t+X$ cross section in the $p_{T}$ range $18 \leq p_{T} \leq 1684 \mathrm{GeV}$ and $|y| \leq 0.5$ in $p_{T}$ bins as chosen by CMS [10] for their measurement. Non-perturbative (NP) corrections for hadronization and multiple parton interactions were estimated by CMS [10] and are published in the Durham HepData project [21]. They are applied to our NLO perturbative QCD predictions. For low- $p_{T}$ jets, the NP corrections are as large as $40 \%$ with a relative uncertainty of $91 \%$ and for large- $p_{T}$ jets they are around $1 \%$ with an error of $0.1 \%$. Theoretical errors from the dependence on the choice of the renormalization scale $\mu_{R}$ and the factorization scale $\mu_{F}$ are determined by varying the scales according to the following combinations of $\xi_{R}$ and $\xi_{F}:(1 / 2,1 / 2),(1 / 2,1),(1,1 / 2)$, $(1,2),(2,1)$ and $(2,2)$, and the largest up and down cross sections for these choices are taken as the scale variation. The default choice is $(1,1)$ with $\mu_{R}=\mu_{F}=p_{T}$. These scale variations modify the prediction of the inclusive jet cross section at the lowest $p_{T}$ bin $(18-21 \mathrm{GeV})$ by $+26.0 \%(-18.7 \%)$, the medium $p_{T}$ bins $(37-43 \mathrm{GeV})$ by $+19.2 \%(-16.5 \%),(97-114$ $\mathrm{GeV})$ by $+15.1 \%(-12.8 \%)$, and the two large $p_{T}$ bins $(174-196 \mathrm{GeV})$ by $+13.1 \%(-11.8 \%)$ and bin $(846-1684 \mathrm{GeV})$ by $+4.4 \%(-13.0 \%)$. The second and fourth value will be used 


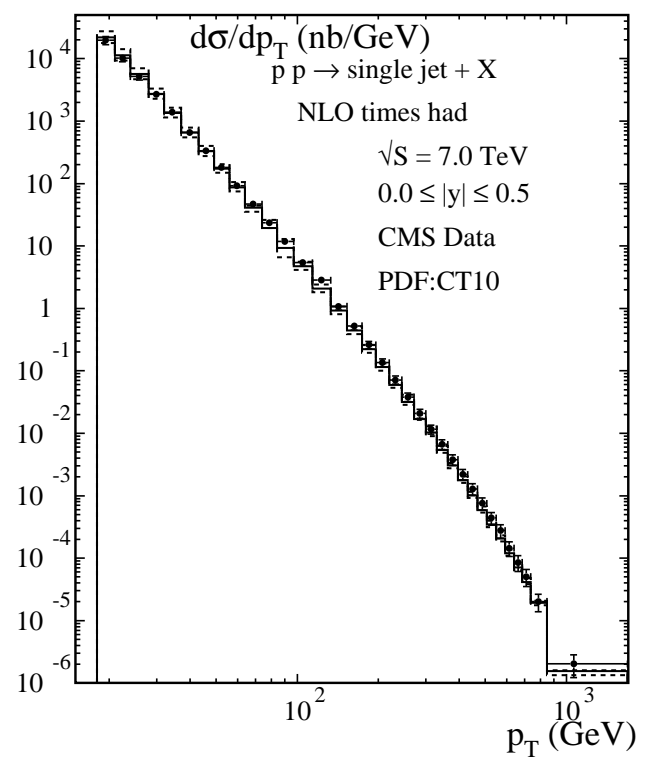

FIG. 1: Single-inclusive jet cross sections $d \sigma / d p_{T}$ as a function of $p_{T}$ compared to the data from CMS [10]. The NLO theoretical predictions are corrected for non-perturbative effects via multiplicative factors. The theoretical error is obtained by independent scale variations given as the dashed curves. The solid curve indicates the default scale choice.

later for estimates of charm-jet cross section errors. The corrected inclusive jet cross section including the theoretical error together with the CMS data [10] is presented in Fig. 1. It shows the jet $p_{T}$ spectra between 18 and $1684 \mathrm{GeV}$. The agreement between data and the theoretical prediction is quite good and quite similar to the comparison shown in the CMS paper [10]. This reference also contains cross section data and a comparison with theory predictions for five more $|y|$ bins up to $|y|=3$, which we have not calculated, exhibiting a similar good agreement between data and pQCD calculations.

The cross section for inclusive charm-jet production is shown in Fig. 2. These cross sections are calculated in $12 p_{T}$ bins for $p_{T}$ between $p_{T}=37 \mathrm{GeV}$ and $p_{T}=196 \mathrm{GeV}$, using the CTEQ6.6M PDF set [18]. The widths of the bins have been chosen according to the CMS publication for b-jet production [17], since so far c-jet production cross sections, which could guide us, have not been measured. The cross sections $d \sigma / d p_{T}$ have been calculated for three rapidity bins: $0.0 \leq|y| \leq 0.5,2.0 \leq|y| \leq 2.2$ and $2.2 \leq|y| \leq 3.2$. The first and second bin are taken as in [17], while the third $|y|$ bin was chosen for the purpose of comparing with cross sections with intrinsic charm PDFs to be considered later. In Fig. 2, 


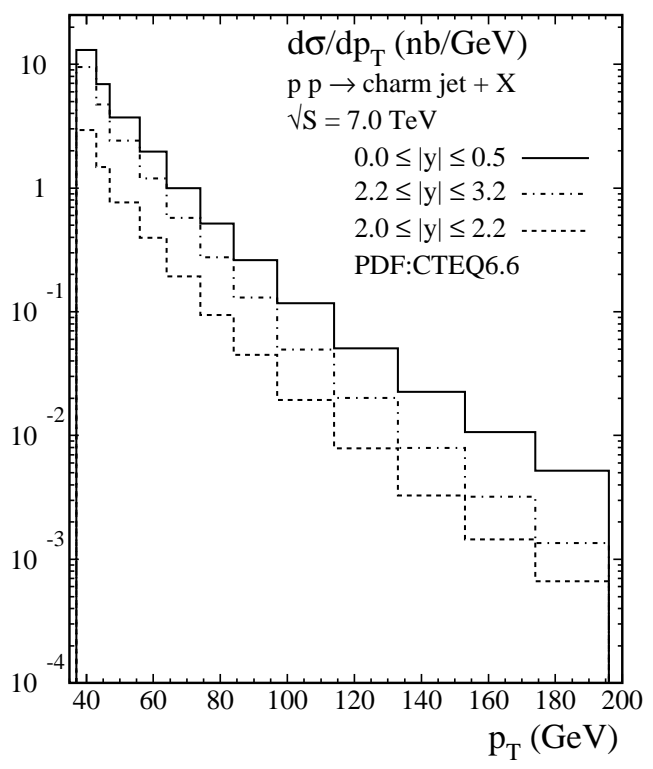

FIG. 2: Cross section $d \sigma / d p_{t}$ for three $|y|$ regions as a function of $p_{T}$ for various bins at larger $p_{T}$.

the largest cross section as a function of $p_{T}$ appears for $|y| \leq 0.5$. The second largest is the one for $2.2 \leq|y| \leq 3.2$, which is larger than the cross section for $2.0 \leq|y| \leq 2.2$ due to the bigger $|y|$-bin size. All cross sections in the three $|y|$ bins have a similar dependence on $p_{T}$. The contribution of the c-jet to the full inclusive jet cross section lies between $1.5 \%$ and $2 \%$, which is shown in Fig. 3, where we have plotted the ratio of the cross sections c-jet/inclusive jet for the three $|y|$ bins. For the $|y|$ bin: $0.0 \leq|y| \leq 0.5$ this ratio is slightly larger than for the other two $|y|$ bins. (For better visibility the ratios for $2.0 \leq|y| \leq 2.2$ and $0.0 \leq|y| \leq 0.5$ are multiplied by factors 10 and 100, respectively). The curves in Fig. 2 and 3 are our main result. We see that the charm cross section in relation to the inclusive jet cross section is rather small. The plots in Fig. 2 and Fig. 3 do not contain non-perturbative corrections for hadronization and multiple parton interactions. If they would be the same as in the inclusive jet cross sections, this would not change the ratio in Fig. 3. In the considered $p_{T}$ range and $|y| \leq 0.5$ these corrections are between $12 \%$ and $3 \%$ (for increasing $p_{T}$ for the bins between $p_{T}=37 \mathrm{GeV}$ and $p_{T}=196 \mathrm{GeV}$ ) in the case of the inclusive jet cross sections [21]. We expect them to be somewhat larger for c-jets than for inclusive jets. However, they have not been calculated yet. Such NP corrections could be estimated using PYTHIA or HERWIG Monte Carlo programs as has been done in the inclusive jet case [10, 21]. Another issue is the selection of the jet events on the experimental and the theoretical side. On the 


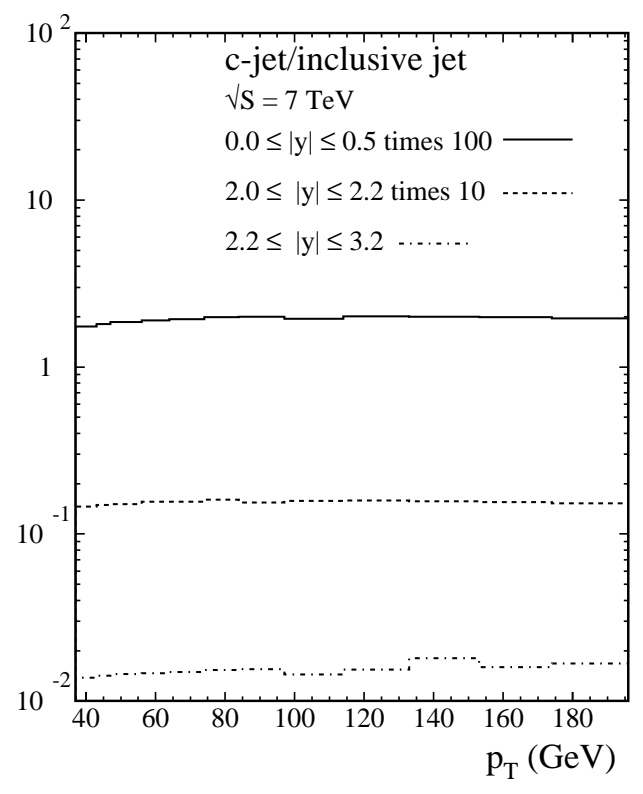

FIG. 3: Ratio of single-inclusive charm-jet cross section and the single-inclusive jet cross section as a function of $p_{T}$ for three rapidity regions $|y|$. The ratios for the lowest two $|y|$ regions are multiplied by 10 and 100 .

experimental side, we assume that the c-jets are identified by finding the secondary decay vertex of the c-hadrons. This means that at least one charmed hadron is in the final state. The inclusive charm-jet cross section $d \sigma / d p_{T}$ contains in addition also a gluon jet or light quark jet, although of course not a light-quark or b-jet instead of the c-jet. For example, already in LO the process $c g \rightarrow c g$ leads to a c-jet and a gluon jet which both contribute to $d \sigma / d p_{T}$ and similarly at NLO. In all common proton PDFs the charm content is generated perturbatively, i.e. by assuming that at threshold near $\mu_{F}=m$ the PDF vanishes or is small and given by higher order contributions while at larger scales $\mu_{F}$ it is determined by evolution. Since many decades it is argued that the charm PDF $c\left(x, \mu_{F}\right)$ at $x>0.1$ could have a non-perturbative intrinsic charm contribution due to the fact that the charm mass is not really large compared to the QCD scale parameter $\Lambda$. In recent work by the CTEQ collaboration, it was investigated how much of the intrinsic charm inside specified models is compatible with the global data samples for particular PDF parametrizations. Such studies were performed starting from the parametrization CTEQ6.5 in [22], starting from CTEQ6.6 in [18] and quite recently starting from CTEQC10 in [23]. In all three investigations [18, 22, 23], two intrinsic charm models have been used: (i) a valence-like 

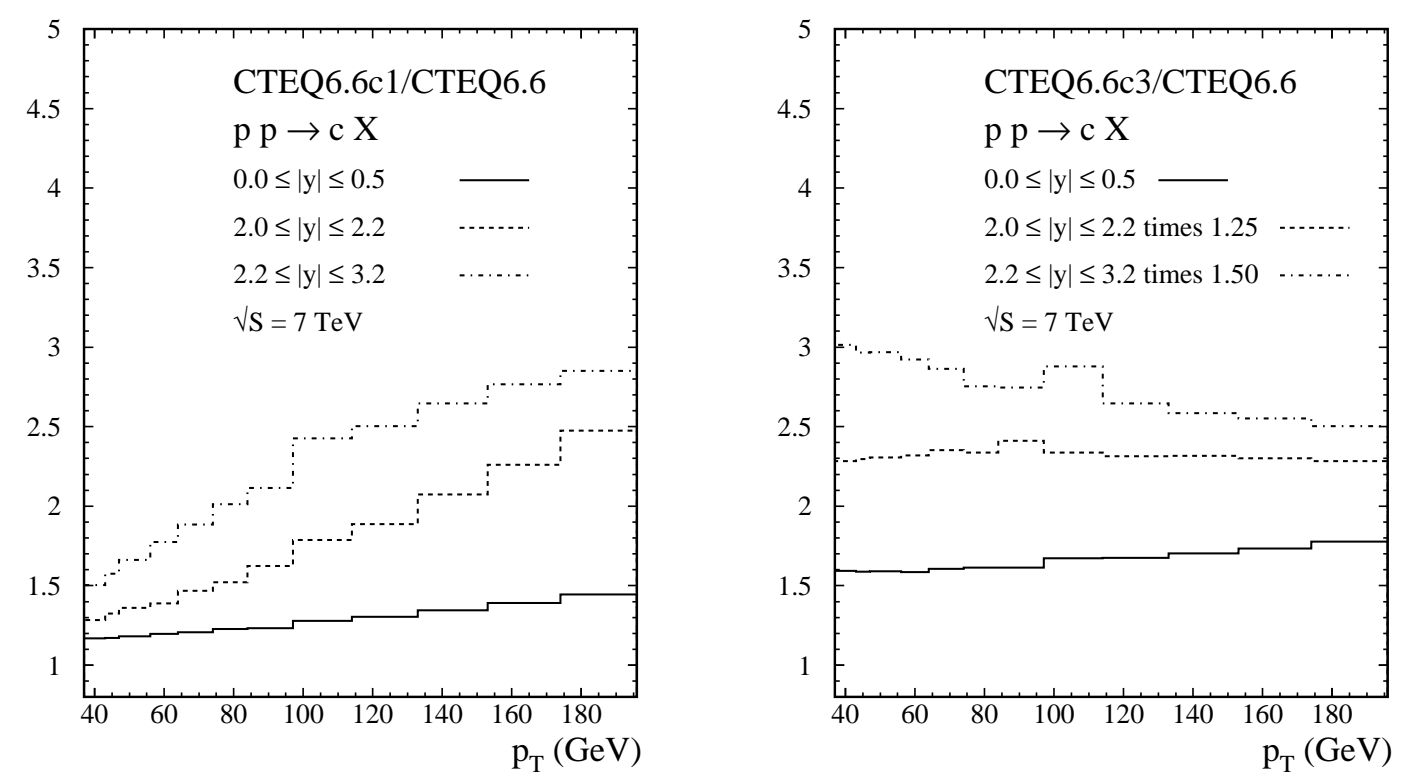

FIG. 4: Ratio of single-inclusive charm-jet cross section for intrinsic charm with BHPS (left-hand side) and SEA (right-hand side) modification to the single-inclusive charm-jet cross section with extrinsic charm only, as a function of $p_{T}$ for three rapidity regions $|y|$.

parton distribution (BHPS model) and (ii) a sea-like parton distribution (SEA model). We select these two models BHPS and SEA, (see [22] for details) with the constraint obtained in the CTEQ analysis [18]. We prefer the corresponding CTEQ6.6c PDF in the program library LHAPDF [24] with a 3.5\% $(c+\bar{c})$ content at the scale $\mu_{F}=1.3 \mathrm{GeV}$ for the BHPS model and the SEA model of high strength. These two particular models for intrinsic charm have been employed also for making predictions for the inclusive production of $D^{0}$ mesons at $\sqrt{S}=7 \mathrm{TeV}$ (to be compared with LHC data) in [6]. From this work it is clear that the effect of intrinsic charm as parametrized by the valence-like model BHPS increases with growing $p_{T}$ and rapidity. For the sea model SEA the effect of intrinsic charm occurred uniformly for all $p_{T}$ and increased with increasing rapidity $y$ [25]. We expect a similar behavior also for charm-jet production. In Fig. 4, we show our results for the relative enhancement of the $p_{T}$-distribution in the bins of $y$ considered previously. The results in Fig. 4 on the left-hand side are for the 3.5\% BHPS model and in Fig. 4 on the right-hand side for the high-strength sea model. For the BHPS model, $p_{T}$ must be large enough to see an increase. For the largest $|y|$ bin: $2.2 \leq|y| \leq 3.2$, one needs $p_{T} \geq 80 \mathrm{GeV}$ to have a rise larger than two. In the case of the lowest $|y|$ bin: $0.0 \leq|y| \leq 0.5$, the enhancement is smaller and increases less with 
growing $p_{T}$. For the sea model plotted on the right-hand side of Fig. 4, the enhancement is nearly independent of $p_{T}$ and $|y|$ bins and varies between 1.6 and 2.0. Hence, apart from the $|y|$ bin: $0.0 \leq|y| \leq 0.5$, it is less than for the BHPS model in the area of high $p_{T}$. For better visibility the curves for $2.0 \leq|y| \leq 2.2$ and $2.2 \leq|y| \leq 3.2$ are multiplied by factors 1.25 and 1.5, respectively. Compared to the inclusive D-meson cross section the increase seems to be similar if one considers the same $p_{T}$ and $|y|$ regions. It is clear that for larger $|y|$ it would be bigger. The question is though, whether these cross sections could be measured with sufficient accuracy.

Of course, these enhancements in the cross sections would be detectable only if they are much larger than any variation of the theoretical cross section due to scale variations. We have not calculated these scale variations for the charm-jet cross sections, but assume that they are very similar to the scale variation of the inclusive jet cross section for the same $p_{T}$ bin. At the largest $p_{T}$ bin in Fig. $4(174-196 \mathrm{GeV})$ the scale variation is $13 \%(-12 \%)$ which is indeed small compared to the enhancement at this $p_{T}$ bin, except for the cross section for the $|y| \leq 0.5$ bin in the case of the valence-like intrinsic charm (see Fig.4 left).

\section{SUMMARY AND CONCLUSIONS}

We have calculated the inclusive charm-jet cross section at NLO of QCD in the zero-mass variable-flavor-number scheme, i.e. with active charm quarks in the proton, for $p p$ collisions

at $\sqrt{S}=7 \mathrm{TeV}$. To test the presence of intrinsic charm contributions in the proton PDF, we have employed two PDF sets which are consistent with global PDF analysis results. We found that they lead to enhancements of the charm-jet cross section at large transverse momentum and rapidity, which might possibly be tested with present and future LHC jet production data.

\section{Acknowledgements}

We thank Michael Klasen and Hubert Spiesberger for advice and fruitful discussions. This work was supported by the German Federal Ministry for Education and Research BMBF through Grant No. 05 H12GUE, by the German Research Foundation DFG through Grant No. KN 365/5-3, and by the Helmholtz Association HGF through Gran No. HA 101. IB 
acknowledges support by the German Science Foundation DFG through the Collaborative Research Centre 676 "Particles, Strings and the Early Universe".

[1] S. Frixione, M.L. Mangano, P. Nason et al., Adv. Ser. Direct. High Energy Phys. 15 (1998) 609 hep-ph/9702287.

[2] M. Klasen and G. Kramer, Eur. Phys. J. C 71 (2011) 1774 arXiv:1104.0095 [hep-ph]].

[3] M. Cacciari and M. Greco, Nucl. Phys. B 421 (1994) 530 hep-ph/9311260].

[4] J. Binnewies, B.A. Kniehl and G. Kramer, Phys. Rev. D 58 (1998) 014014 hep-ph/9712482 and earlier papers quoted there.

[5] B. A. Kniehl, G. Kramer, I. Schienbein and H. Spiesberger, Phys. Rev. D 71 (2005) 014018 hep-ph/0410289 and earlier papers quoted there.

[6] B.A. Kniehl, G. Kramer, I. Schienbein and H. Spiesberger, Eur. Phys. J. C 72 (2012) 2082 arXiv:1202.0439 [hep-ph]].

[7] B. Abelev et al. [ALICE Collaboration], JHEP 1201 (2012) 128 arXiv:1111.1553 [hep-ex]].

[8] The ATLAS Collaboration, ATL-PHYS-PUB-2011-012; ATLAS-CONF-2011-017.

[9] R. Aaij et al. [LHCb Collaboration], Nucl. Phys. B 871 (2013) 1 [arXiv:1302.2864 [hep-ex]].

[10] S. Chatrchyan et al. [CMS Collaboration], Phys. Rev. Lett. 107 (2011) 132001 arXiv:1106.0208 [hep-ex]].

[11] G. Aad et al. [ATLAS Collaboration], Phys. Rev. D 86 (2012) 014022 arXiv:1112.6297 [hepex]].

[12] M. Klasen and G. Kramer, Z. Phys. C 72 (1996) 107 hep-ph/9511405]; ibid. C 76 (1997) 67 hep-ph/9611450; M. Klasen, T. Kleinwort and G. Kramer, Eur. Phys. J. direct C 1 (1998) 1 hep-ph/9712256.

[13] M. Klasen, Rev. Mod. Phys. 74 (2002) 1221 hep-ph/0206169].

[14] Z. Nagy, Phys. Rev. Lett. 88 (2002) 122003 hep-ph/0110315; Z. Nagy, Phys. Rev. D 68 (2003) 094002 hep-ph/0307268.

[15] T. Kluge, K. Rabbertz and M. Wobisch in Proceedings of the 14th International Workshop on Deep Inelastic Scattering (DIS 2006), Tsukuba, Japan 2006 (World Scientific, Singapore, 2007) p. 483, hep-ph/0609285.

[16] H.-L. Lai et al., Phys. Rev. D 82 (2010) 074024 arXiv:1007.2241 [hep-ph]]. 
[17] S. Chatrchyan et al. [CMS Collaboration], JHEP 1204 (2012) 084 [arXiv:1202.4617 [hep-ex]].

[18] P. M. Nadolsky, H.-L. Lai, Q.-H. Cao, J. Huston, J. Pumplin, D. Stump, W.-K. Tung and C. P. Yuan, Phys. Rev. D 78 (2008) 013004 [arXiv:0802.0007 [hep-ph]].

[19] S. Catani et al., Nucl. Phys. B 406 (1993) 187; S.D. Ellis and D.E. Soper, Phys. Rev. D 48 (1993) 3160 hep-ph/9305266.

[20] M. Cacciari, G.P. Salam and G. Soyez, JHEP 0804 (2008) 063 [arXiv:0802.1189 [hep-ph]].

[21] http://hepdata.cedar.ac.uk/view/ins902309.

[22] J. Pumplin, H. L. Lai and W. K. Tung, Phys. Rev. D 75 (2007) 054029 hep-ph/0701220].

[23] S. Dulat et al., Phys. Rev. D 89 (2014) 073004 [arXiv:1309.0025 [hep-ph]].

[24] LHAPDF (The Les Houches Accord PDF Interface), http://projects.hepforge.org/lhapdf/pdfsets.

[25] See also: B. A. Kniehl, G. Kramer, I. Schienbein and H. Spiesberger, Phys. Rev. D 79 (2009) 094009 arXiv:0901.4130 [hep-ph]]. 